\title{
PREFERÊNCIA DE Palmistichus elaeisis POR PUPAS DE DIFERENTES LEPIDÓPTEROS PRAGA ${ }^{1}$
}

\author{
Maria Aparecida Leão Bittencourt²; Evoneo Berti Filho ${ }^{3 *}$ \\ ${ }^{2}$ Pós-Graduanda do Depto. de Entomologia, Fitopatologia e Zoologia Agrícola - ESALQ/USP. \\ ${ }^{3}$ Depto. de Entomologia, Fitopatologia e Zoologia Agrícola - ESALQ/USP, C.P. 9 - CEP: 13418-900 - Piracicaba, SP. \\ *e-mail: eberti@carpa.ciagri.usp.br
}

RESUMO: Palmistichus elaeisis Delvare \& LaSalle, 1993 (Hym., Eulophidae), uma espécie de endoparasitóide foi coletado em pupas de Sabulodes sp. (Lep., Geometridae), em área de eucalipto. Foram testadas quatro espécies de lepidópteros-praga: Diatraea saccharalis (Fabricius, 1794) (Crambidae), Anticarsia gemmatalis Hübner, 1818, Heliothis virescens (Fabricius, 1781) e Spodoptera frugiperda (J.E. Smith, 1797) (Noctuidae), com o objetivo de estudar a preferência para oviposição, visando a criação massal do parasitóide. Os resultados mostraram que a porcentagem de parasitismo variou de $90 \%(H$. virescens) a $100 \%$. Em $D$. saccharalis, o parasitóide $P$. elaeisis apresentou a maior razão sexual $(0,98)$ e em $H$. virescens a menor $(0,69)$. A espécie de hospedeiro influenciou significativamente o tamanho dos machos de $P$. elaeisis (1,64 mm em S. frugiperda e 1,49 $\mathrm{mm}$ em $H$. virescens), mas não o das fêmeas. $O$ sexo do hospedeiro é importante no total de parasitóides produzidos, enquanto que as espécies do hospedeiro não interferiram no total de parasitóides produzidos.

Palavras-chave: Palmistichus elaeisis, Insecta, Eulophidae, controle biológico

\section{PREFERENCE OF Palmistichus elaeisis FOR PUPAE OF SOME LEPIDOPTEROUS PESTS}

\begin{abstract}
Palmistichus elaeisis Delvare \& LaSalle, 1993 (Hym.: Eulophidae) was found parasitizing pupae of Sabulodes sp. (Lep.: Geometridae) in an Eucalyptus plantation. To study the preference of this parasitoid for pupae of other lepidopteran pests, an experiment was conducted testing the following Lepidoptera species: Diatraea saccharalis (Fabricius, 1794) (Crambidae), Anticarsia gemmatalis Hübner, 1818, Heliothis virescens (Fabricius, 1781) and Spodoptera frugiperda (J.E. Smith, 1797) (Noctuidae). The percentage of parasitism ranged from $90 \%$ ( $H$. virescens) to $100 \%$. The highest value for the parasitoid sexual ratio was 0.98 for $D$. saccharalis, and the lowest was 0.69 for $H$. virescens. The host species significantly affected the size of $P$. elaeisis males $(1.64 \mathrm{~mm}$ in $S$. frugiperda and $1.49 \mathrm{~mm}$ in $H$. virescens), and not that of females. The sex of the host had significant effect on the total number of parasitoids, whereas the host species had no statistical effect on the same variable.
\end{abstract}

Key words: Palmistichus elaeisis, insecta, Eulophidae, biological control

\section{INTRODUÇÃO}

Entre os insetos entomófagos, a Ordem Hymenoptera é dominante em número, e nos casos de sucesso no controle biológico mais de $2 / 3$ foram conseguidos com himenópteros parasíticos. Os calcidóideos são um dos grupos mais importantes no controle biológico em floresta (Berti Filho, 1985).

Delvare \& LaSalle (1993) descreveram um novo gênero de Tetrastichinae da Região
Neotropical, nele incluindo uma nova espécie denominada Palmistichus elaeisis. Le Verdier \& Genty (1988) verificaram que o controle natural de Hispoleptis subfasciata Pic (Col.: Chrysomelidae), praga desfolhadora de Elaeis guineensis Jacq. (dendezeiro), era realizado por um eulofídeo que atacava os ovos da praga, sendo este identificado posteriormente como $P$. elaeisis.

O objetivo deste trabalho foi avaliar a preferência para oviposição de $P$. elaeisis

$\overline{{ }^{1} \text { Parte da Tese de }}$ Doutorado do primeiro autor apresentada à ESALQ/USP - Piracicaba, SP. 
Delvare \& LaSalle, 1993 (Hym.: Eulophidae) em pupas de diferentes lepidópteros-praga: Diatraea saccharalis (Fabricius, 1794) (Crambidae), Anticarsia gemmatalis Hübner, 1818, Heliothis virescens (Fabricius, 1781) e Spodoptera frugiperda (J.E. Smith, 1797) (Noctuidae), visando a criação massal do parasitóide.

\section{MATERIAL E MÉTODOS}

Esta pesquisa foi conduzida no Laboratório de Controle Biológico do Departamento de Entomologia da Escola Superior de Agricultura "Luiz de Queiroz", da Universidade de São Paulo, em Piracicaba, SP. O parasitóide $P$. elaeisis foi coletado em pupas de Sabulodes sp. (Lep., Geometridae), na região de Itatinga (SP), em eucaliptal pertencente à Ripasa Papel e Celulose, e mantido no laboratório em gaiolas de madeira $(70 \times 50 \times 40 \mathrm{~cm}) \mathrm{com}$ tela na lateral e vidro na metade superior da parte frontal, a $25 \pm 1^{\circ} \mathrm{C}, 70 \pm 10 \%$ de UR e $14 \mathrm{~h}$ de fotofase. Os adultos foram alimentados com solução de mel a $10 \%$.

Avaliaram-se quatro espécies de lepidópteros, criados em dieta artificial, como hospedeiros: $D$. saccharalis, $A$. gemmatalis, $H$. virescens e $S$. frugiperda. Dez pupas de cada espécie, com 24 a 48 horas de idade, foram expostas por 24 horas em uma mesma gaiola contendo $P$. elaeisis emergidos no dia. Os pesos médios dos hospedeiros foram: $A$. gemmatalis $(q=0,24 \mathrm{~g}$ e $\delta=0,29 \mathrm{~g}), D$. saccharalis $(q=0,18 \mathrm{~g})$, $H$. virescens $(q=0,27 \mathrm{~g}$ e $\delta=0,30 \mathrm{~g})$ e $S$. frugiperda $(q=0,28 \mathrm{~g}$ e $\delta=0,28 \mathrm{~g})$. Em relação ao sexo do hospedeiro, as proporções variaram: somente pupas $\&$ (D. saccharalis), $40 \%$ de $q$ e $60 \%$ de $\delta$ (A. gemmatalis), $50 \%$ de $\%$ e $50 \%$ de бे (S. frugiperda) e $60 \%$ de $\$$ e $40 \%$ de $\delta$ ( $H$. virescens). Após 24 horas de exposição aos parasitóides, os hospedeiros foram individualizados em tubos de vidro $(8,5 \times 2,5 \mathrm{~cm})$ e colocados em câmaras climáticas a $25 \pm 1^{\circ} \mathrm{C}, 70$ $\pm 10 \%$ de UR e fotofase de 14 horas.

Os seguintes parâmetros foram avaliados: porcentagem de parasitismo, número de parasitóides emergidos e razão sexual. Usouse as seguintes terminologias: pupas parasitadas, referindo-se às pupas inviabilizadas pelo parasitóide o qual não emergiu, e pupas com parasitóides, referindo-se às pupas de onde emergiram parasitóides. Os dados obtidos para o total de parasitóides foram analisados através do módulo LAB do sistema SAS - Statistical Analisis System. Utilizou-se os procedimentos
PROC FREQ (teste de Qui-Quadrado) e PROC NPAR1WAY (teste de Wilcoxon) para análise e comparação de médias a nível de significância de $5 \%$.

Avaliou-se o tamanho dos parasitóides emergidos por hospedeiro, sendo medidos 10 fêmeas e 10 machos de cada um. Os resultados foram submetidos a análise de variância e as médias comparadas pelo teste de Tukey ao nível de $5 \%$.

\section{RESULTADOS E DISCUSSÃO}

A porcentagem de parasitismo (pupas parasitadas + pupas com parasitóides) variou de $90 \%$ para $H$. virescens, a $100 \%$ para os demais hospedeiros (Figura 1).

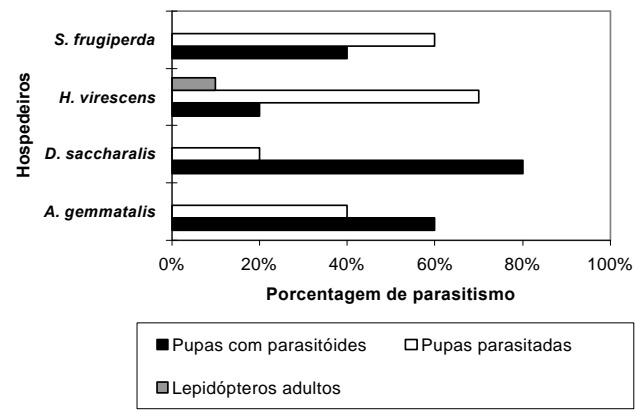

Figura 1- Parasitismo de Palmistichus elaeisis em livre escolha. Temperatura: $25 \pm 1^{\circ} \mathrm{C}$; UR: $70 \pm 10 \%$; fotofase: $14 \mathrm{~h}$.

O número de parasitóides emergidos, a razão sexual e o tamanho médio de fêmeas e machos obtidos foram resumidos na TABELA 1.

Em relação ao sexo dos hospedeiros, a análise estatística mostrou que houve diferença significativa $\left(\chi^{2}=0,02\right.$ e Prob $\left.>\chi^{2}=0,88\right)$ no total de parasitóides emergidos, sendo que esta diferença pode estar relacionada ao número de pupas utilizadas (pupas fêmea $=25$ e pupas macho=15) ou ao valor nutricional de cada hospedeiro. Vinson \& Barbosa (1987) relatam que uma vez parasitado, o hospedeiro passa a ser a fonte de alimento e 0 abrigo dos endoparasitóides, podendo a qualidade do hospedeiro ser um fator importante na determinação do tamanho, sexo e sobrevivência do parasitóide.

A análise estatística mostrou que não houve diferença significativa entre os hospedeiros $\left(\chi^{2}=5,77\right.$ e Prob $\left.>\chi^{2}=0,12\right)$ em relação ao total de parasitóides emergidos. 
TABELA 1 - Média de parasitóides ( \pm EP), razão sexual e tamanho de indivíduos de Palmistichus elaeisis que emergiram de diferentes hospedeiros. Temperatura: $25 \pm 1^{\circ} \mathrm{C}$; UR: $70 \pm 10 \%$; fotofase: $14 \mathrm{~h}$.

\begin{tabular}{lccccc}
\hline & $\begin{array}{c}\text { Parasitóides } \\
\text { emergidos de } \\
\text { pupas } q\end{array}$ & $\begin{array}{c}\text { Parasitóides } \\
\text { emergidos de } \\
\text { pupas o }\end{array}$ & $\begin{array}{c}\text { Razão } \\
\text { Sexual }\end{array}$ & $\begin{array}{c}\text { Fêmeas } \\
(\mathrm{mm})^{1}\end{array}$ & $\begin{array}{c}\text { Machos } \\
(\mathrm{mm})^{1}\end{array}$ \\
\hline A. gemmatalis & $9,0 \pm 18,9$ & $19,5 \pm 19,5$ & 0,89 & $2,23 \pm 0,02 \mathrm{a}$ & $1,54 \pm 0,02 \mathrm{ab}$ \\
D. saccharalis & $22,4 \pm 10,5$ & - & 0,98 & $2,25 \pm 0,01 \mathrm{a}$ & $1,59 \pm 0,03 \mathrm{ab}$ \\
H. virescens & 0 & $19,2 \pm 37,9$ & 0,69 & $2,21 \pm 0,02 \mathrm{a}$ & $1,49 \pm 0,02 \mathrm{~b}$ \\
S. frugiperda & $17,6 \pm 30,3$ & $5,4 \pm 9,7$ & 0,89 & $2,21 \pm 0,03 \mathrm{a}$ & $1,64 \pm 0,04$ a \\
\hline
\end{tabular}

${ }^{1}$ Médias seguidas pela mesma letra não diferem entre si pelo teste de Tukey a $5 \%$.

Não houve diferença estatística entre os hospedeiros quanto ao tamanho das fêmeas de $P$. elaeisis, mas houve diferença estatística no tamanho dos machos. Sugere-se que os parasitóides machos oriundos de $S$. frugiperda, por apresentarem maior tamanho, poderiam ser mais facilmente escolhidos pelas fêmeas na hora da cópula, o que refletiria numa característica importante em relação ao hospedeiro.

\section{CONCLUSÕES}

- Todos os hospedeiros testados ( $A$. gemmatalis $D$. saccharalis, $H$. virescens e Spodoptera frugiperda) possibilitam a criação de $P$. elaeisis.

- A espécie do hospedeiro interfere significativamente no tamanho dos machos de $P$. elaeisis.

- O sexo do hospedeiro é importante no total de parasitóides.

- Não houve preferência para oviposição de $P$. elaeisis nos hospedeiros testados.

\section{REFERÊNCIAS BIBLIOGRÁFICAS}

BERTI FILHO, E. O parasitismo no controle integrado de pragas florestais. Silvicultura, v.10, n.39, p.7-10, 1985.

DELVARE, G.; LaSALLE, J. A new genus of Tetrastichinae (Hymenoptera: Eulophidae) from the Neotropical Region, with the description of a new species parasitic on key pests of oil palm. Journal of Natural History, v.27, p.435-444, 1993.

LE VERDIER, A.; GENTY, P. Dégâts et contrôle de Hispoleptis subfasciata Pic. Oléagineux, v.43, n.11, p.397-407, 1988.

VINSON, S.B.; BARBOSA, P. Interrelationships of nutritional ecology of parasitoids. In: SLANSKY JR., F.; RODRIGUES, J.G. (Ed.) Nutritional ecology of insects, mites, spiders, and related invertebrates. New York: John Wiley, 1987. cap.21, p.673-695.

Recebido para publicação em 28.01.99

Aceito para publicação em 13.09 .99 